See discussions, stats, and author profiles for this publication at: https://www.researchgate.net/publication/321629291

\title{
Bubble nucleation from micro-crevices in a shear flow
}

Article in Experiments in Fluids - January 2018

DOI: 10.1007/s00348-017-2459-y

5 authors, including:

$$
\text { Tim F. Groß }
$$

Technische Universität Darmstadt

15 PUBLICATIONS 55 CITATIONS

SEE PROFILE

-2) Peter Franz Pelz

1 Technische Universität Darmstadt 179 PUBLICATIONS 358 CITATIONS

SEE PROFILE

Some of the authors of this publication are also working on these related projects:

Intensification studies View project

Project free surface flow and power extraction View project 


\title{
Bubble nucleation from micro-crevices in a shear flow
}

\section{Experimental determination of nucleation rates and surface nuclei growth}

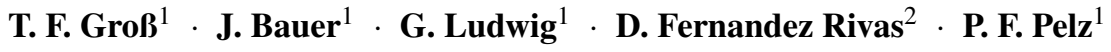

Received: date / Accepted: date

\begin{abstract}
The formation of gas bubbles at gas cavities located in walls bounding the flow occurs in many technical applications but is usually hard to observe. Even though, the presence of a fluid flow undoubtedly affects the formation of bubbles, there are very few studies that take this fact into account. In the present paper new experimental results on bubble formation (diffusion-driven nucleation) from surface nuclei in a shear flow are presented. The observed gas-filled cavities are micrometre-sized blind holes etched in silicon substrates. We measure the frequency of bubble generation (nucleation rate), the size of the detaching bubbles and analyse the growth of the surface nuclei. The experimental findings support an extended understanding of bubble formation as a self-excited cyclic process and can serve as validation data for analytical and numerical models.
\end{abstract}

Keywords cavitation · nucleation · degassing · bubble formation

\section{Introduction}

Investigations on the formation of gas bubbles in supersaturated liquids are of interest in many research fields and for different technical applications (Jones et al (1999a)). Cavitation in hydraulic machines or on ship propellers (Brennen (1995)), degassing of the working fluid in oil hydraulic devices (Freudigmann et al (2017)), bubbles in carbonated beverages (Liger-Belair (2004)) or the decompression sickness of scuba divers (Neumann (2002)) are only examples

E-mail: Peter F. Pelz

peter.pelz@fst.tu-darmstadt.de

${ }^{1}$ Chair of Fluid Systems, TU Darmstadt,

64287 Darmstadt, Germany

2 Mesoscale Chemical Systems group, University of Twente

7500 AE Enschede, The Netherlands for the importance of bubble formation in liquids. In the mentioned cases the formation of bubbles is usually not desired. There are other research fields and applications in which bubble formation is desired, e.g. the cleaning of surfaces (Verhaagen and Fernandez Rivas (2015); BolanosJimenez et al (2017)), the removal of bacteria and germs in water (Sarc et al (2016)), the fragmentation of kidney stones in the human body by acoustic cavitation called lithotripsy, the targeted release of pharmacological agents (both Brennen (2015)) and the enhancement of the efficiency of mixing processes (Spiridonov (2015)).

Recent studies about cavitating nuclei on hydrophobic surfaces (Bremond et al (2005); Borkent et al (2009)), skinstabilised surface nuclei (Andersen and Mørch (2015)), the activation of roughness elements that serve as nucleation spots (van Rijsbergen and van Terwisga (2011)), vapourbubble nucleation in Rayleigh-Bénard turbulence (Guzman et al (2016)), vapour bubbles and the role of permanent gas (Prosperetti (2017)), oscillations of gas-pockets in crevices (Gelderblom et al (2012)), electrolysis-driven and pressurecontrolled diffusive growth of bubbles on microstructured surfaces (van der Linde et al (2017)) and the field of nanobubbles (Lohse and Zhang (2015)) underline the importance of research in the broad field of bubbles and bubble formation.

The formation of gas bubbles in a supersaturated liquid is usually referred to as bubble nucleation. The classical nucleation theory (homogeneous nucleation) describes the formation of gas bubbles within the liquid bulk in the absence of a pre-existing gas phase due to the thermal motion of molecules (Brennen (1995)). Heterogeneous nucleation denotes bubble formation at weak spots in the liquid, i.e. surfaces, particles, gas bubbles or gas entrapped in cavities (Brennen (1995)). If the pressure is far above the vapour pressure of the condensed fluid, the most important physical quantity for bubble formation is the supersaturation of 
the liquid, $\zeta:=c / c_{\mathrm{S}}-1$ with gas concentration of the noncondensable gas $c$ and saturation concentration $c_{\mathrm{S}}$. Homogeneous nucleation only occurs in case of large supersaturations, $\zeta \gg 100$, since a nucleation energy barrier has to be overcome to create the new phase. Homogeneous nucleation is empirically unachievable since heterogeneous nucleation would occur at the walls of the test section or the container already at smaller supersaturations. Thus, homogeneous nucleation is irrelevant for water at normal temperatures (Brennen (1995)). It is common sense that bubble formation in liquids is caused by heterogeneous nucleation, in particular the growth of pre-existing gas cavities. In acoustic cavitation the crevice model of bubble nucleation of Atchley and Prosperetti (1989) is commonly accepted. This theory neglects the influence of gas diffusion so that it does not apply for bubble formation in supersaturated solutions.

In many flow situations one observes very pronounced bubble formation at pre-existing gas cavities. In these cases, the formation of bubbles is a result of mass transfer, more precisely molecular diffusion, cf. figure 1 and Groß and Pelz (2017). These gas cavities are referred to as surface nuclei. Surface nuclei grow due to mass transfer of noncondensable gas and eventually free bubbles detach. After the detachment the process repeats and a periodical production of bubbles can be observed. The process lasts as long as the liquid is supersaturated, a fact known to everyone in the context of carbonated beverages. In technical flows there is always "new" liquid flowing past the surface nuclei keeping the process running over a long time unless the surface nuclei are deactivated somehow (Borkent et al (2009)). Bubble formation at surface nuclei can be interpreted as self-excited cyclic process triggered by the bubble detachment, cf. figure 1. We call this process diffusion-driven nucleation to distinguish it clearly from homogeneous and heterogeneous nucleation. It is reasonable to divide the process into two parts, i) the growth of surface nuclei and ii) the detachment of free bubbles (Groß et al (2016); Groß and Pelz (2017)).

To gain insights into the field of diffusion-driven nucleation it is worthwhile to study the growth of dispersed bubbles and then apply the findings to surface nuclei. Epstein and Plesset (1950) provided a solution of Fick's second law for a spherical bubble growing or shrinking in an supersaturated or undersaturated quiescent liquid and estimated the occurring mass flux. Scriven (1959) provided a more general analysis by considering the influence of the radial expansion of the bubble. Both papers report a linear dependence of the squared bubble radius on time, $R_{\mathrm{B}}^{2} \propto t$. Jones et al (1999b) applied this theory to the growth of surface nuclei in a quiescent liquid and confirmed the validity of the relation experimentally. In recent works the so called history effect is considered (Peñas-López et al (2016, 2017)). Following this theory the diffusion mass flux is a function of the time history of the concentration at the bubble boundary.
In addition, the detachment of bubbles leads to a depletion of the gas concentration in the surrounding liquid near to the nucleation spot (Moreno Soto et al (2016)).

In most technical applications, a fluid flow affects the evolution of the concentration field and thus the mass transfer. Parkin and Kermeen (1963) investigated the growth of gas bubbles in the boundary layer of a submerged body. The mass transfer is strongly intensified due to the relative motion of the bubble to the flowing liquid. van Wijngaarden (1967) applied the theory to cavitating flows and expanded it by considering surface tension and vapour pressure. Lochiel and Calderbank (1964) provide a comprehensive study of mass transfer across liquid-gas interfaces of bubbles exposed to a fluid flow. They studied various bubble shapes (spheres, oblate and prolate spheroids) as well as various boundary conditions (mobile, immobile and rapidly moving interfaces). In addition to growing bubbles it is useful to study the shrinkage of bubbles in undersaturated liquids. Nüllig and Peters (2013) investigated the influence of the rising velocity of bubbles on their shrinkage behaviour in undersaturated water experimentally. The experiments indicate a linear dependence of the bubble radius on time, $R_{\mathrm{B}} \propto t$.

In addition to surface nuclei growth, the detachment of bubbles is of importance to understand the process of diffusion-driven nucleation from surface nuclei. In a quiescent liquid, the buoyancy force has to overcome the capillary force to cause a bubble detachment from a gas cavity (Jones et al (1999a)). The critical diameter for bubble detachment is denoted as Fritz diameter (Fritz (1935)) sometimes. In a fluid flow, dynamic forces act on the bubble and cause an earlier detachment resulting in smaller bubble sizes. Duhar and Colin (2006) investigated bubble detachment from wall orifices in shear flows with wall shear rates of $\dot{\gamma}<11 \mathrm{~s}^{-1}$. The experiments showed that the diameters of the detaching bubbles were influenced by the viscous shear flow only to a small extent. This finding is not surprising since the shear rates were relatively small and the buoyancy force is still the dominating force in these experiments. Investigations on bubble detachment from wall-orifices in liquid cross-flows (Nahra and Kamonati (2003)) and bubble detachment from surfaces in the important field of flow boiling (Chen et al (2012)) underline the importance of this topic in other research fields.

Peters and Honza (2014) were the first to systematically study diffusion-driven nucleation from surface nuclei using a laminar radial gap flow and silicone oil as liquid. The supersaturation of the liquid was given by the spatial pressure distribution in the flow. Blind holes with diameters of $d=0.6 \mathrm{~mm}$ and $0.8 \mathrm{~mm}$ drilled in steel worked as nucleation sites. The ratio of blind hole diameter to gap height was between 3 and 16. Thus, the detachment of bubbles was strongly influenced by the narrow gap. Nucleation rates of 

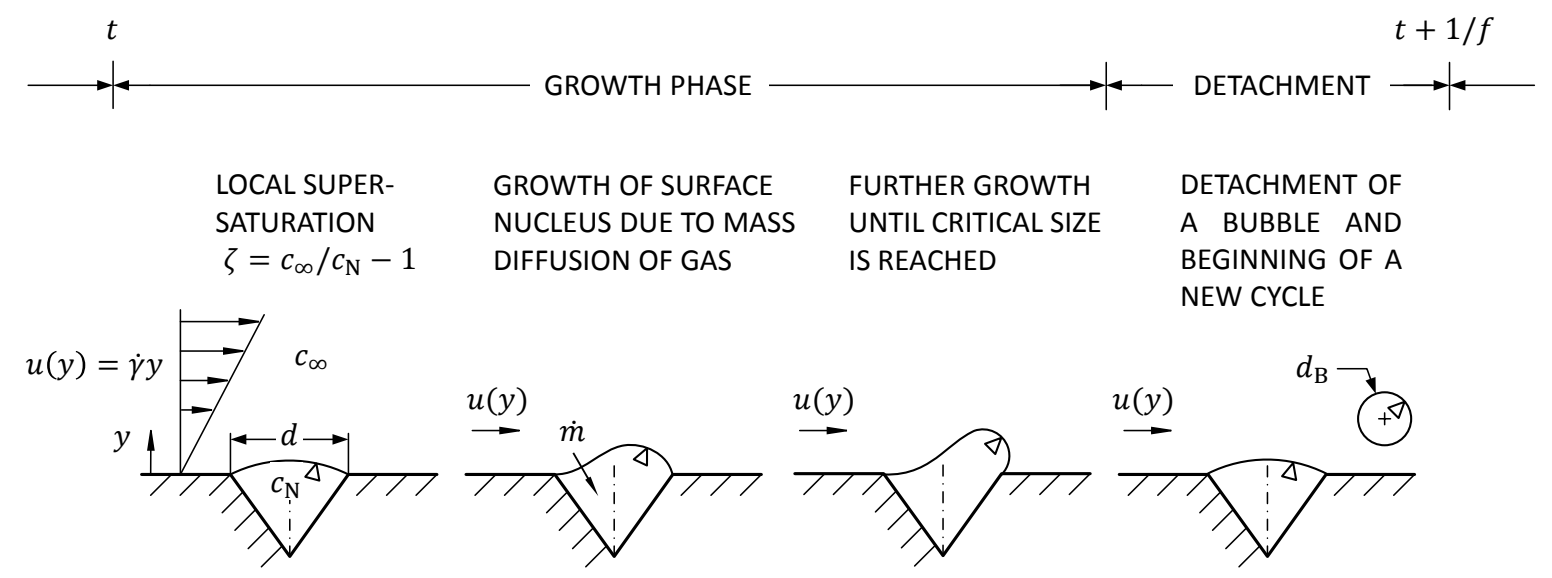

Fig. 1 Principle sketch of the growth of a surface nucleus and the detachment of a free nucleus in a shear flow, cf. Groß and Pelz (2017).

the order of magnitude of $10^{-2} \mathrm{~Hz}$ to $10^{1} \mathrm{~Hz}$ were measured. The authors found that the nucleation rate depends on the shear rate at the wall but could not identify a functional dependence. Shear rates in the order of magnitude of $10^{2} \mathrm{~s}^{-1}$ to $10^{3} \mathrm{~s}^{-1}$ were realised. In Groß et al (2015) some of the authors of this paper continued the work of Peters and Honza (2014) and modified the experimental setup to conduct experiments with larger shear rates and achieved nucleation rates of up to $1 \mathrm{kHz}$. In Groß et al (2016) a channel with rectangular cross-section was used to study the bubble detachment. It was found that in some cases the bubble detachment is the result of a Plateau-Rayleigh instability, a phenomenon responsible for the breakup of liquid jets (Rayleigh (1879); Groß and Pelz (2017)).

In Groß and Pelz (2017) two of the authors of this paper provide a theoretical in depth analysis of the nucleation rate considering the influence of the shear rate, the supersaturation of the liquid and the crevice diameters. Based on the simple model $f=\dot{m} / m_{\mathrm{B}}$ (nucleation rate $f$, diffusion mass flux $\dot{m}$, mass of the detaching bubbles $m_{\mathrm{B}}$ ) it was shown that the shear rate influences both the mass flux of gas that diffuses into the surface nucleus and the detachment process of the bubbles. In dimensionless arguments the dimensionless nucleation rate depends on Péclet number, Weber number, supersaturation of the liquid and the dimensionless gas solubility of the liquid.

In the present paper, the experimental setup used by Groß et al (2016) is further developed to conduct experiments with water. The diameters of the observed crevices (pits in a silicon substrate) are $25 \mu \mathrm{m}, 50 \mu \mathrm{m}$ and $100 \mu \mathrm{m}$ and thus at least one order of magnitude smaller than crevices used in previous studies. The ratio of crevice diameter to gap height is always smaller than $5.6 \times 10^{-2}$. As we will see in section 3 the nucleation process is not influenced by the height of the gap. The wall shear rates are of the order of magnitude of $10^{2} \mathrm{~s}^{-1}$ to $10^{3} \mathrm{~s}^{-1}$. The experiments indicate that the nucleation rate and the volume of the detaching bubbles show a non-linear dependence on the shear rate at the wall. The experiments also show that in some cases a linear dependency of the equivalent radius of the growing surface nucleus on time, $R \propto t$, can be found.

As far as we know, the present paper contains the most comprehensive experimental study of diffusion-driven nucleation from surface nuclei in a fluid flow. It is the first time that nucleation spots on a microscale are considered. The presented results can serve as validation data for axiomatic models, cf. Groß and Pelz (2017), or numerical simulations which are needed to correctly consider bubble formation in technical applications such as pumps, valves, oil hydraulic components as well as microfluidic devices.

The paper can be outlined as follows. In section 2 we describe the experimental set-up and the experimental procedure. In section 3 we show experimental results for the measurement of (i) nucleation rates, (ii) size of the detaching bubbles, and (iii) growth of surface nuclei and discuss the most important findings. In section 4 we discuss the growth of surface nuclei in detail and provide an interpretation of the results based on boundary layer theory. The paper closes with a summary of the main results in section 5 .

\section{Experimental setup and procedure}

Figure 2 shows a sketch of the experimental setup which is operated as a batch process. A pressure difference between two tanks drives the flow. The tanks have a volume of 50 litres each and are connected through a piping system that contains control valves and the test section. The test section is a rectangular channel in an acrylic glass housing, cf. figure 3. We measure the pressure in the tanks and in the test section (Keller Series 33X), flow rate (Fisher \& Porter D10D) 


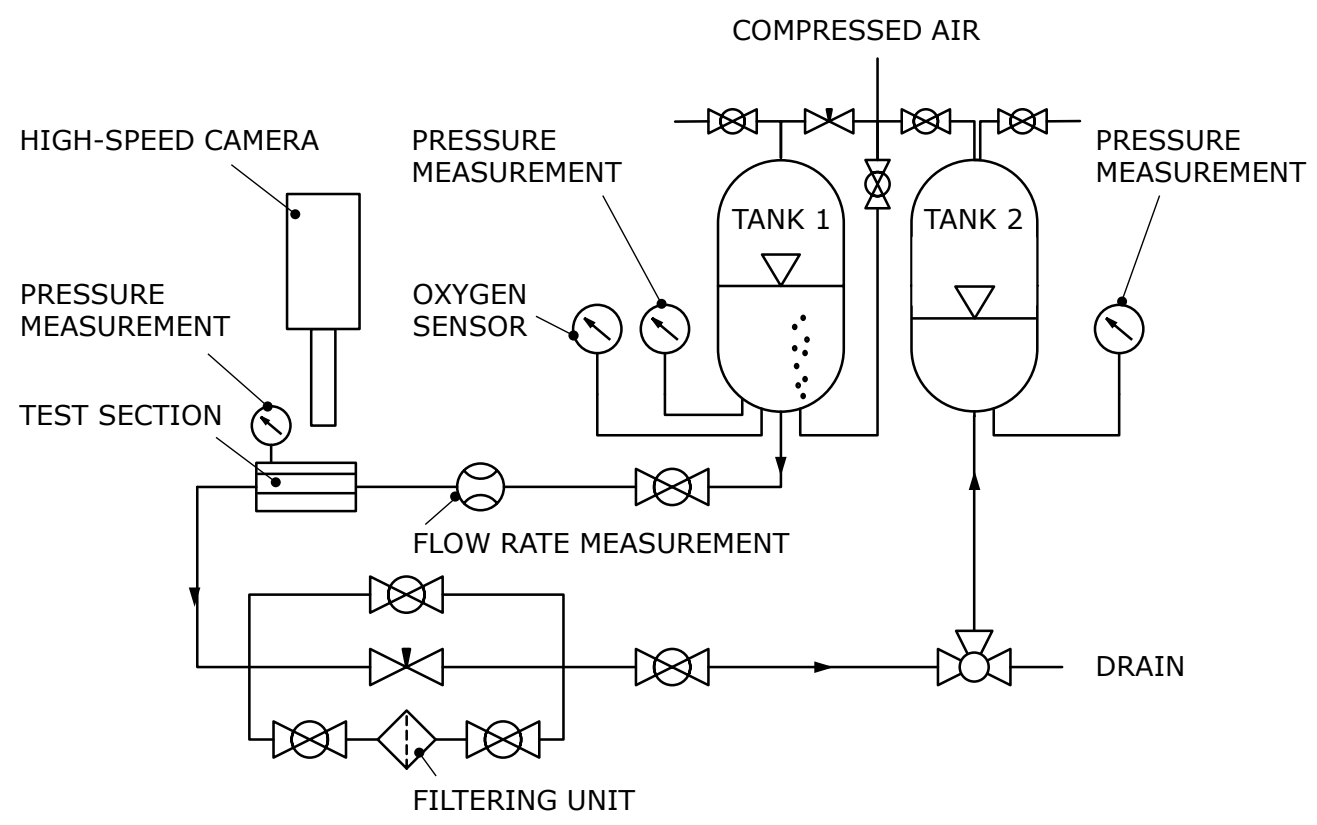

Fig. 2 Sketch of experimental setup.

as well as the oxygen content and the temperature in tank 1 (Hamilton VisiFerm DO Arc 120). The nucleation process is visualised using a high-speed camera system (IDT Motion Pro Y7 S3), a long distance microscope (Optem Fusion 125) and stroboscopic lighting (Veritas Constellation 120) which is synchronised with the camera system. By adjusting the pressure difference between the two tanks and using a needle valve the flow rate can be controlled manually in a wide range. Maximum flow rates of up to $101 / \mathrm{min}$ can be achieved. By closing the control valve the flow rate can be set to zero.

To study the nucleation process, a supersaturation of the liquid is required. Therefore, compressed air is injected at the bottom of tank 1 to reach a specific gas saturation in the liquid before starting the experiments. The gas saturation cannot be measured directly. We measure the oxygen content and assume that it is representative for all solved non-condensable gases. Indeed, this is reliable if both the pressure in tank 1 and the oxygen content are constant for a sufficiently long time, i.e. 10 to 15 minutes. To create a supersaturation at the nucleation sites, the saturation pressure in tank 1 has to be higher than the operating pressure in the test section. According to Henry's law (Henry (1803)) the supersaturation can be calculated with $\zeta:=p_{1} / p-1$ where $p_{1}$ is the saturation pressure in tank 1 and $p$ is the pressure in the test section.

It has to be ensured that there are only a few bubbles dispersed in the liquid before it reaches the test section. Even though the degassing in the piping upstream of the test section cannot be measured directly, the amount of free gas visible in the test section allows an approximate estimation.
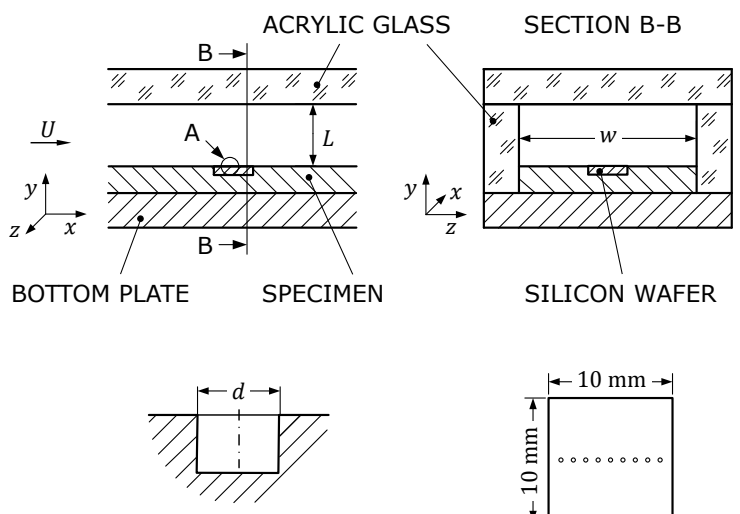

DETAIL A

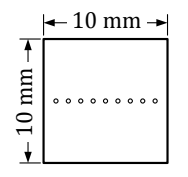

SILICON WAFER

Fig. 3 Sketch of test section.

Besides degassing of the liquid free gas might enter the system through sealings or connecting elements which are not hermetically airtight. Usually there are only a few bubbles dispersed in the liquid so that a constant supersaturation can be assumed. However, the degassing of the liquid practically limits both the supersaturation and the flow rate. The operation point is set by controlling the pressure in tank 1 and the flow rate by adjusting the control valves manually. During the measurements the pressure in the test section is kept constant at 1.47 bar. The supersaturation $\zeta$ is varied between 0.1 to 1 . Wall shear rates in the range of $\dot{\gamma} \sim 10^{2} \mathrm{~s}^{-1}$ to $10^{3} \mathrm{~s}^{-1}$ are realised.

The test section is a channel with rectangular cross section, cf. figure 3 . It has a width of $w=30 \mathrm{~mm}$. The height 


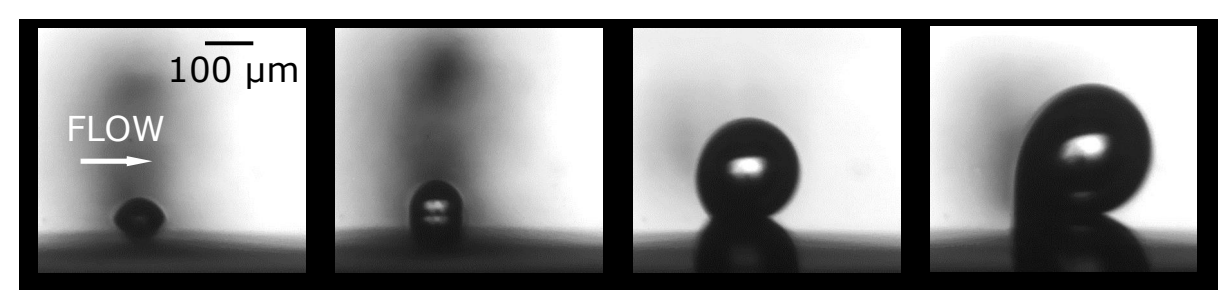

Fig. 4 Growth of a bubble at a nucleus with $100 \mu \mathrm{m}$ in a flow with $\dot{\gamma}=340 \mathrm{~s}^{-1}$. The time interval between the images is $7 \mathrm{~s}$. The nucleation rate is $4.8 \times 10^{-2} \mathrm{~Hz}$.

of the channel $h$ can be varied between $1.8 \mathrm{~mm}$ and $3.8 \mathrm{~mm}$ in discrete steps of $0.5 \mathrm{~mm}$. The shear rate at the wall is $\dot{\gamma}=6 Q /\left(h^{2} w\right)$ with flow rate $Q$ considering a laminar flow. We never observed any discontinuities in our experimental results so that we consider the flow to be laminar even for higher shear rates which could be a crucial assumption but works well with the experiments. In case of a turbulent flow, the wall-shear rate would also be the relevant kinematic quantity if the crevice diameter is smaller than the viscous length $l_{v}:=v / \sqrt{\tau_{\mathrm{w}} / \rho}$ with viscosity $v$ and density $\rho$ of the fluid and wall-shear stress $\tau_{\mathrm{w}}$.

The test section is made of acrylic glass to ensure optical accessibility from top and side view perspective. The nucleation process is studied at micrometre-sized pits which are etched in silicon substrates with dimensions $10 \mathrm{~mm} \times$ $10 \mathrm{~mm} \times 0.5 \mathrm{~mm}$. The silicon substrates are attached to a removable holding system of stainless steel which is placed in the test section. To study the nucleation process we use three different pit diameters: $25 \mu \mathrm{m}, 50 \mu \mathrm{m}$ and $100 \mu \mathrm{m}$. The pits are approximately $20 \mu \mathrm{m}$ deep. See Fernandez Rivas et al (2010) and Zijlstra et al (2015) for further information about the production of the pits.

The nucleation process is visualised using a high-speed camera system with a resolution of 1920 x 1080 pixels and a maximum frame rate of 10,600 frames per second. Usually a frame rate of 50 to 200 frames per second is sufficient for the visualisation of the nucleation processes. To visualise the crevices and the detaching bubbles it is necessary to use a long-distance microscope. Stroboscopes which are synchronised with the frequency of the camera are used to ensure sufficient lighting. In our experiments we measure the nucleation rate and the size of the detaching bubbles and furthermore evaluate the growth process. The nucleation rate is measured from top view perspective. Bubble size and growth behaviour are measured from side view perspective. Since the depth of focus of the long distance microscope is only a few micrometres, recordings from side view perspective are accompanied by time-consuming adjustment work. Figure 4 shows a typical recording of surface nuclei growth from side view perspective.

In contrast to experiments in quiescent liquids it is much more challenging to keep the liquid free of contaminations in experiments with flowing liquids. Tanks, pipes, hoses, sealings and connecting elements are not always easy to clean. Usually the amount of liquid used is larger in experiments with flowing liquids compared to experiments with quiescent liquids. The detachment process can be influenced by contaminations that adhere to the substrate. Micrometresized dust and dirt particles deposit on the surface or, even worse, in the crevices even though the liquid is cleaned by passing a submicro filtering unit. Besides that dust particles in the air can adhere to the surface of the substrate during conversion work of the test section, e.g. while changing the substrate or gap height, when the test section is not filled with liquid. Besides cleaning of the surface one additionally has to dry the pores so that air can be entrapped in the micro-crevices by exposing the substrate to the liquid in the test section. The entrapment works as described by Bankoff (1958). Air is entrapped in the crevices when the liquid spreads over the silicon substrate and thus over the crevices. This works so well, that nearly every crevice contains a surface nucleus after the re-filling of the test section. Care must be taken to ensure that the liquid is not undersaturated after the re-filling because otherwise the entrapped gas diffuses into the surrounding liquid.

\section{Experiments}

In the experiments we varied shear rate, gap height, crevice diameter and the supersaturation of the liquid. In total we evaluated about 300 operation points. We measured i) the nucleation rate, ii) the size of the detaching bubbles, and iii) the time dependent growth of surface nuclei.

The measurement of the nucleation rate is conducted from top-view perspective. It is not necessary to visualize exact outlines of the surface nucleus to identify the nucleation rate with sufficient accuracy. In contrast, the growth measurements which are done from side-view perspective are much more time-consuming than the nucleation rate measurements because of the necessary adjustment of the camera and the long-distance microscope due to the limited depth of field of the optical system and the limited optical resolution. For this reason, not all data points of the nucleation rate measurements shown in figure 5 are present in the results of the volume measurements in figure 7 . In addition, we have limited ourselves to the two crevice diameters 
$d=25 \mu \mathrm{m}$ and $d=100 \mu \mathrm{m}$ in the size and growth measurements. For better clarity, figure 8 shows only a few cases of surface nuclei growth.

It should be noted that the measurements were conducted in measurement series. One measurement series is characterised by one crevice diameter and one gap height. After a measurement series the setup is partly dismantled, the gap height is varied and/or a new substrate is mounted. This requires a drainage of the water and a re-filling of the test section prior to the start of the next measuring series. Even though shear rate, gap height and supersaturation of the liquid can be controlled or measured the entrapment of gas in the crevices is a random process which cannot be influenced. Large fluctuations occurring in the bubble volume measurements are expression of the sensitivity of bubble nucleation from micro-crevices in a fluid flow. Nevertheless, we found that the individual measurements at specific crevices in specific operation points show a very constant nucleation rate which varies over time only to a small extent. In this point diffusion-driven nucleation from microcrevices in fluid flows equals diffusion-driven nucleation in quiescent liquids, e.g. in carbonated beverages.

\subsection{Nucleation rate}

Figure 5 shows measurements of the the nucleation rate (frequency of bubble production) and its dependence on the shear rate for the three crevice diameters $d=25 \mu \mathrm{m}, 50 \mu \mathrm{m}$, and $100 \mu \mathrm{m}$. In Groß et al (2016) and Groß and Pelz (2017) it has been shown both theoretically and experimentally that the nucleation rate is proportional to the supersaturation $\zeta$ of the liquid. We therefore plot the reduced nucleation rate $f / \zeta$ against the shear rate to improve clarity and significance. Different symbols in a graph mark different gap heights that have been evaluated. The measuring error of the frequency is estimated to be smaller than $15 \%$ of the measured values. For better clarity, error bars are not shown.

Figures 5 a-c clearly show that the reduced nucleation rate increases with increasing shear rate. We find a nonlinear dependence of the reduced nucleation rate on the shear rate of the form $f / \zeta \propto \dot{\gamma}^{\alpha}$. It is not possible to identify an exponent $\alpha$ that is generally valid for all three diameters. The graphs indicate that the exponent increases with increasing crevice diameter. We find the three relations $f / \zeta \propto \dot{\gamma}^{0.98}$, $f / \zeta \propto \dot{\gamma}^{1.64}$ and $f / \zeta \propto \dot{\gamma}^{1.70}$.

In addition, the variation of the gap height allows to conclude that it does not influence the bubble formation in our experiments. This is not surprising since the ratio of crevice diameter to gap height is always smaller than $5.6 \times 10^{-2}$. Thus, the detaching bubbles are very small compared to the height of the gap. A change of the gap height at a constant flow rate is already considered in the shear rate.

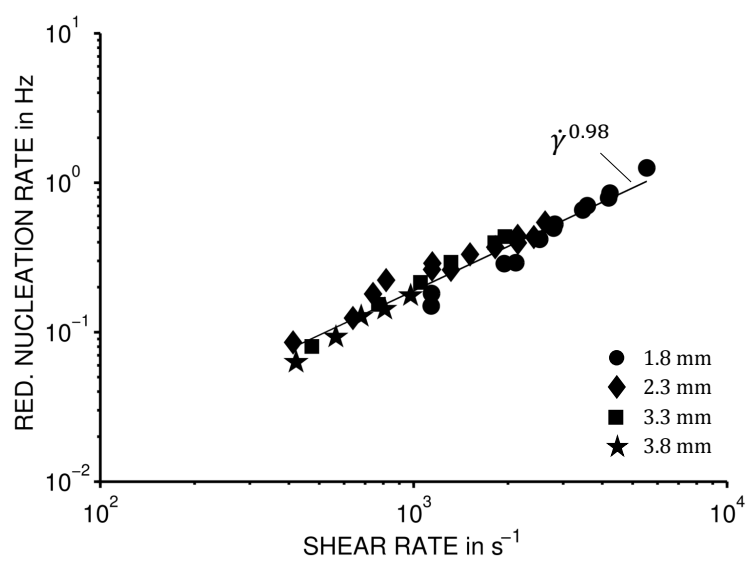

(a) $d=25 \mu \mathrm{m}$

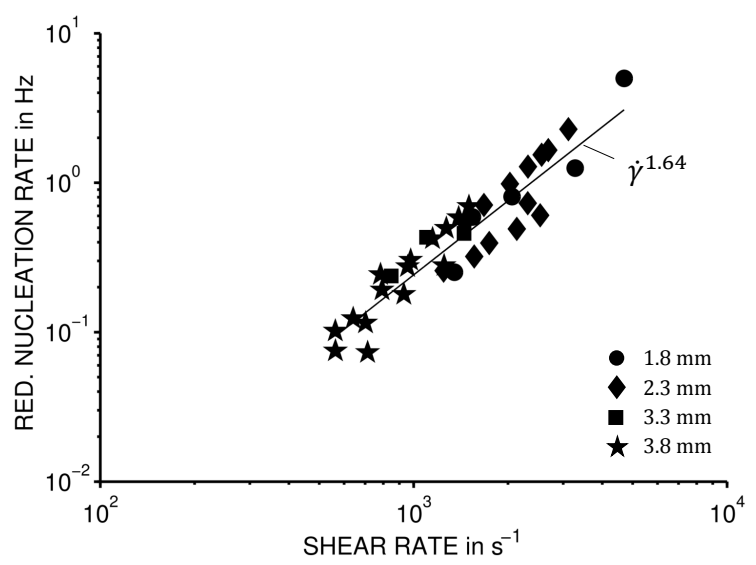

(b) $d=50 \mu \mathrm{m}$

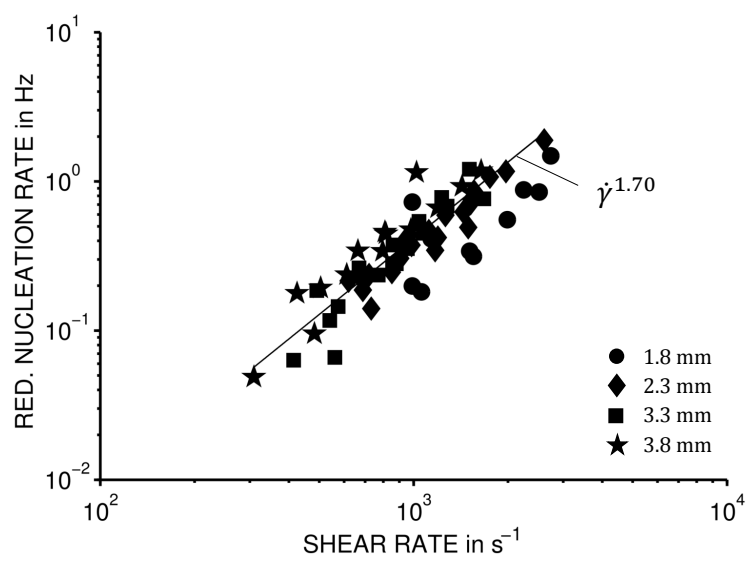

(c) $d=100 \mu \mathrm{m}$

Fig. 5 a - c) Reduced nucleation rate $f / \zeta$ vs. shear rate at the wall for different crevice diameters $d=25 \mu \mathrm{m}, 50 \mu \mathrm{m}$, and $100 \mu \mathrm{m}$ and different gap heights, $1.8 \mathrm{~mm}, 2.3 \mathrm{~mm}, 3.3 \mathrm{~mm}$, and $3.8 \mathrm{~mm}$. 


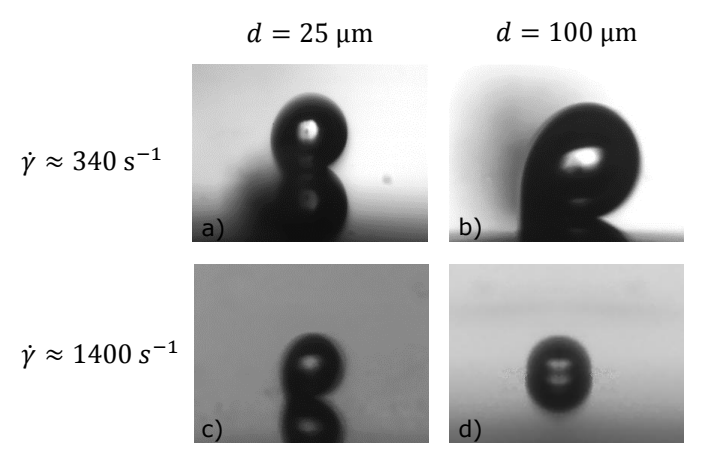

Fig. 6 Shape of surface nucleus prior to bubble detachment for the crevice diameters $25 \mu \mathrm{m}$ and $100 \mu \mathrm{m}$ and two shear rates. The pictures for different crevice diameters are not scaled.

As discussed before, the exponent of the power law $f \propto \dot{\gamma}^{\alpha}$ differs substantially depending on the crevice diameter. So far, the reason for this large discrepancy is not doubtlessly clarified. Figure 6 shows the shape of surface nuclei prior to the detachment of a bubble for two crevice diameters and two shear rates. With increasing shear rate, the bubble volume decreases which will be discussed in the next section. It can be seen that the shape of the surface nuclei for $d=25 \mu \mathrm{m}$ does not vary fundamentally for the increased shear rate. For $d=100 \mu \mathrm{m}$ the surface nucleus shows a clearly visible shape change. Please note, that the surface nucleus in picture b) looks very similar to the one shown in picture c). Hence, we can assume that in case of smaller crevices way larger shear rates are needed to achieve a hemispherical shape. Even though we do not know exactly how the nucleation rate is related to the shape of the surface nucleus, a dependency can be assumed. To solve this issue nucleation rate measurements in a wider range of shear rates have to be conducted.

\subsection{Volume of detaching bubbles}

In the current setup it is not possible to measure the size of the bubbles after the detachment because of motion blur. We therefore measure the size of the surface nuclei immediately prior to detachment and assume that the size of the surface nuclei that visibly protrudes into the liquid equals the size of the detached bubbles. This of course is a crucial assumption when a part of the surface nucleus not visible from side view perspective is part of the detaching bubble. However, this is a straightforward approach to determine the bubble volume.

Large measuring uncertainties $(30-40 \%$ of the bubble volume) have to be considered. The major uncertainties are the limitation of the optical resolution and the fact that the surface nuclei are always in motion immediately prior to the detachment. Besides that one has to keep in mind that the bubble volume is determined using a two-dimensional projection of the surface nucleus. Deformations in the third di-

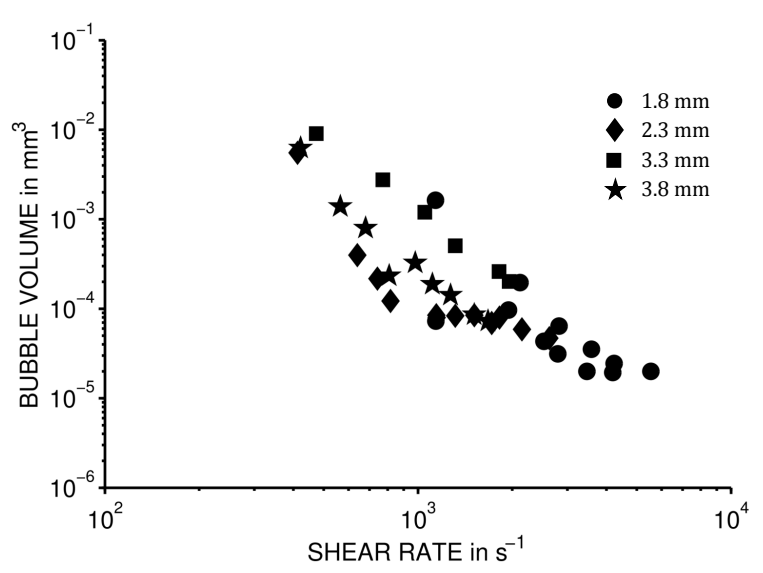

(a) $d=25 \mu \mathrm{m}$

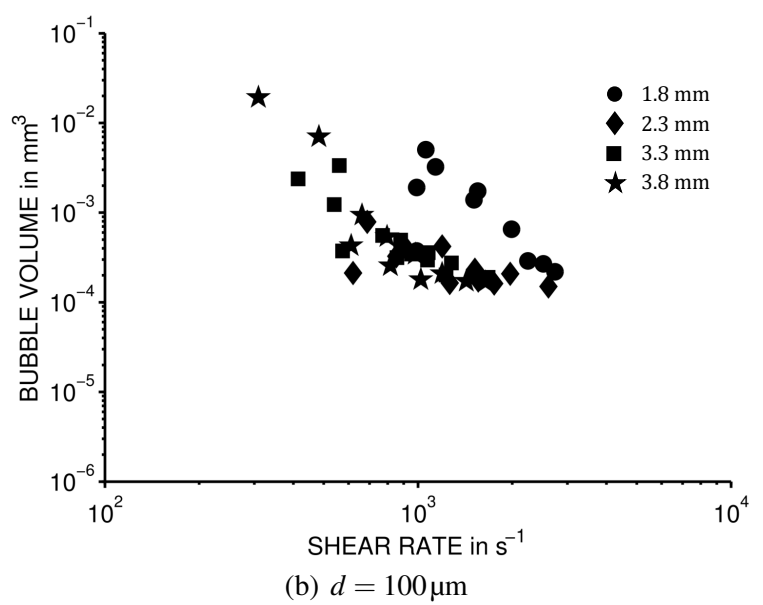

Fig. 7 a - b) Bubble volume vs. shear rate at the wall for crevice diameters $d=25 \mu \mathrm{m}$ and $100 \mu \mathrm{m}$ and different gap heights, $1.8 \mathrm{~mm}, 2.3 \mathrm{~mm}$, $3.3 \mathrm{~mm}$, and $3.8 \mathrm{~mm}$.

mension can not be considered. If the surface nucleus is not spherical, cf. right image of figure 4 or figure $6 \mathrm{~b}$ ), the volume is determined by integrating the radius along the main axis of the deformed surface nucleus. While interpreting the shown results the large measurement uncertainties must be kept in mind. For better clarity of the graphs, error bars are not shown.

Figure $7 \mathrm{a}$-b shows the bubble volume $V_{\mathrm{B}}$ plotted against the shear rate for the diameters $d=25 \mu \mathrm{m}$ and $100 \mu \mathrm{m}$ and different gap heights. As one expects, the bubble volume decreases with increasing shear rate but it is hardly possible to identify a power law as seen for the nucleate rate measurements. It should be noted, that the volume of a hemispherical surface nucleus which diameter equals the diameter of the crevice is $4.1 \times 10^{-6} \mathrm{~mm}^{3}$ for $d=25 \mu \mathrm{m}$ and $2.6 \times 10^{-4} \mathrm{~mm}^{3}$ for a $d=100 \mu \mathrm{m}$. As shown in figure 6 , the surface nuclei are way larger than a hemisphere for $d=25 \mu \mathrm{m}$ and more or less hemispherically for a wide range of shear rates for $d=100 \mu \mathrm{m}$ 
Figure 7 a shows a large scattering of the experimental results especially for small shear rates and thus for large surface nuclei. This proves that the experiments presented are very sensitive. It also indicates that the determination of bubble volumes on the small length scale is very difficult. In figure $7 \mathrm{~b}$ it is possible to find at least two datasets, i.e. different gap heights, that clearly can be distinguished from each other (circles vs. other symbols).

In contrast to shear rate, gap height and supersaturation of the liquid it is not possible to influence how the surface nuclei are entrapped in the crevice. It might be the case that a surface nucleus is positioned in the crevice in a different way each time the crevice is filled with air at the beginning of a new measuring series. At first sight, it seems that the surface nuclei are attached to the edge of the crevices prior to bubble detachment, cf. figures 4 and 6 . After bubble detachment the surface nuclei have to be attached to the side walls or the bottom of the crevice since it is not completely filled with air anymore. The way the surface nucleus is positioned in the crevice and the way it protrudes into the liquid during its growth process could explain the clearly visible discrepancy of different measurement series. These effects could be summed up as local factors that have to be examined in future studies. As far as we know, this issue is not completely understood even for the no-flow case, cf. Liger-Belair (2005) who observed that the bubble formation in carbonated beverages (champagne) strongly depends on the size and shape of the pre-existing micro-cavities. On the one hand, the microcrevices used in this study are well-defined by the production process. On the other hand, we do not known how the microscopic structure of the edge of the crevices influences the detachment process. In future studies new experimental methods have to be found to take a closer look on the detachment process.

\subsection{Growth of surface nuclei}

As mentioned above, figure 4 shows a typical sequence of the growth of a surface nucleus at a crevice with diameter $d=100 \mu \mathrm{m}$. In the first two frames the surfaces nucleus reaches a hemispherical shape. As the surface nucleus grows, it is forced in the direction of the flow and finally a bubble detaches (not shown in the sequence). By evaluating the volume of the surface nucleus frame by frame, it is possible to study the growth of the surface nuclei depending on time.

In figure 8 a-b the equivalent radii $R$ of surface nuclei are plotted versus time $t$ for different crevice diameters $d=25 \mu \mathrm{m}$ and $100 \mu \mathrm{m}$ and different shear rates. The radii are calculated with $R=(3 V /(4 \pi))^{1 / 3}$ with $V$ being the time dependent volume of the surface nuclei protruding into the liquid. Thus, $R$ is the radius of a spherical bubble

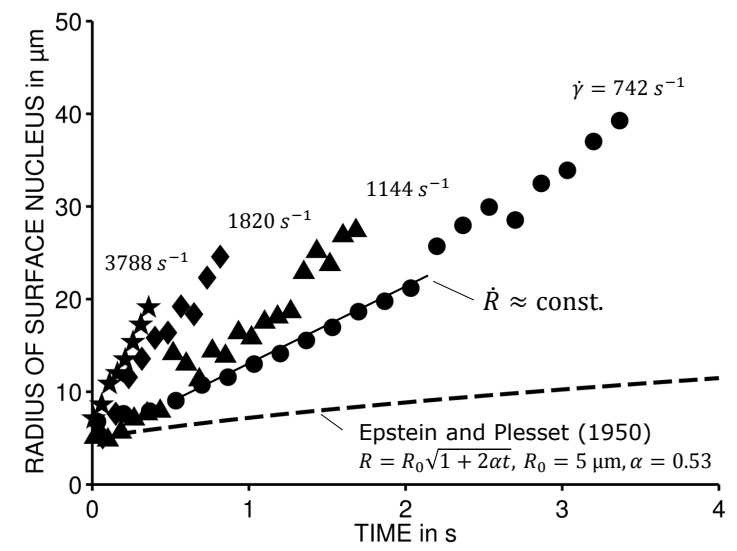

(a) $d=25 \mu \mathrm{m}$

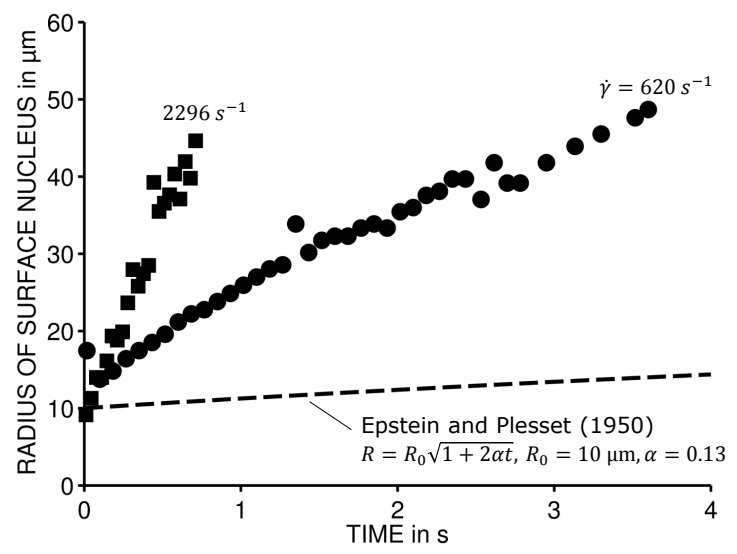

(b) $d=100 \mu \mathrm{m}$

Fig. 8 a - b) Equivalent surface nucleus radius vs. time for different crevice diameters $d=25 \mu \mathrm{m}$ and $100 \mu \mathrm{m}$ and shear rates. The dashed lines show the no-flow solution for the growth of a sphere, cf. Epstein and Plesset (1950). Here $\alpha=\mathscr{D} \Lambda \zeta /\left(R_{0}^{2}(\zeta+1)\right)$ with diffusion coefficient $\mathscr{D}=2 \times 10^{-9} \mathrm{~m}^{2} / \mathrm{s}, \Lambda=0.02$ and $\zeta=0.5$.

which volume equals the volume of the corresponding surface nucleus. This approach allows a comparison of surface nuclei of different shapes and sizes. The results can also be compared with the no-flow solution of Epstein and Plesset (1950) for the growth of a spherical bubble (dashed line). Please note, that it is also possible to draw a corresponding volume vs. time plot which contains redundant information. An evaluation of the radius of curvature could also be useful as long as the surface nuclei are not deformed strongly.

Both graphs of figure 8 indicate that the growth rate $\dot{R}=$ $\mathrm{d} R / \mathrm{d} t$ increases with increasing shear rate which one would expect. In some cases, e.g. $d=25 \mu \mathrm{m}$ and $\dot{\gamma}=3788 \mathrm{~s}^{-1}$, the growth rate $\dot{R}$ seems to be constant which implies $R \propto t$. In other cases, e.g. $d=100 \mu \mathrm{m}$ and $\dot{\gamma}=620 \mathrm{~s}^{-1}$, the growth rate decreases with increasing radius. In the following we provide a theoretical interpretation of these results. 


\section{Nuclei growth, analytical assessment}

The results presented in the previous section indicate that there is a linear dependence of the equivalent radius of the surface nucleus on time or a slight decrease of the growth rate $\dot{R}$ with increasing radius. The former indicates that the volume of the surface nucleus growth with $t^{3}$.

As mentioned in section 1, a linear dependence for radius growth has also been found for ascending spherical bubbles that shrink in an undersaturated liquid, cf. Nüllig and Peters (2013). To shed some light on this we take a look at the diffusion mass flux $\dot{m}$ into the spherical bubble with radius $R_{\mathrm{B}}$ and volume $V_{\mathrm{B}} \propto R_{\mathrm{B}}^{3}$. When $\rho$ denotes the gas density within the bubble one obtains $\dot{m}=\rho \mathrm{d} V_{\mathrm{B}} / \mathrm{d} t \propto \rho R_{\mathrm{B}}^{2} \dot{R}_{\mathrm{B}}$ for the diffusion mass flux. For $\dot{R}_{\mathrm{B}}=$ const. the surface specific mass flux $\dot{m} / R_{\mathrm{B}}^{2}$ is constant as well.

We now adapt this approach to the growth of surface nuclei in a shear flow in terms of the equivalent radius $R$. It should be noted, that this approach is especially valid for $R \gg d$ but it is also useful for the interpretation of cases that do not fulfil this assumption.

The question arises if the finding $\dot{R}=$ const. is consistent with theoretical considerations about the diffusion mass flux in a fluid flow. The diffusion mass flux depends on the velocity field with its characteristic velocity $U$, characteristic length $l$, diffusion coefficient $\mathscr{D}$, concentration difference $\Delta c$, and molar mass $M$. Using dimensional analysis one finds that the relation $\dot{m}=\mathrm{fn}(U, l, \mathscr{D}, \Delta c, M)$ can be reduced to $S h=\mathrm{fn}(P e)$ with $S h:=\dot{m} /(\mathscr{D} M \Delta c l)$ and $P e:=U l / \mathscr{D}$, cf. Groß and Pelz (2017).

We now consider the gas-liquid interface to be plane which of course is a crucial assumption in case of large surface nuclei but has been successfully applied to spherical bubbles before, cf. Parkin and Kermeen (1963) or van Wijngaarden (1967). Depending on the velocity field, boundary layer theory provides two solutions for the advectiondiffusion problem. For a uniform flow with velocity $U$ one obtains the solution $S h \propto(U R / \mathscr{D})^{1 / 2}$ with $R$ being the characteristic length, cf. Lochiel and Calderbank (1964). In case of a shear flow with shear rate at the wall $\dot{\gamma}$ one obtains $S h \propto\left(\dot{\gamma} R^{2} / \mathscr{D}\right)^{1 / 3}$, cf. Groß and Pelz (2017).

In our experiments the surface nucleus is exposed to a shear flow. If the surface nucleus is small and only a small part protrudes into the liquid, the velocity at the liquid-gas interface is approaching zero. With increasing radius of the surface nucleus the mean velocity increases. It is reasonable using $U \sim \dot{\gamma} R$ for larger surface nuclei. Using the relation $S h \propto(U l / \mathscr{D})^{1 / 2}$ yields $\dot{m} / R^{2}=$ const. and thus $R \propto t$. This is what is indeed measured and shown in figure 8 .

The proposed solution of the boundary layer theory implies that there is no velocity gradient (uniform velocity field) at the liquid-gas interface which is a crucial assumption. The boundary layer solution for a shear flow, $S h \propto$ $\left(\dot{\gamma} R^{2} / \mathscr{D}\right)^{1 / 3}$, leads to the solution $\dot{m} / R^{2} \propto R^{-1 / 3}$ and thus $R \propto t^{3 / 4}$. The two approaches based on boundary layer theory lead to reasonable results. It might be the case that there is a transition from $S h \propto P e^{1 / 3}$ to $S h \propto P e^{1 / 2}$ during bubble growth. Note that the relation $R \propto t^{1 / 2}$ has been found for the no-flow case, Epstein and Plesset (1950).

\section{Conclusions}

Bubble formation in supersaturated liquids is an important issue in many technical applications and different research fields. Even though bubble nucleation has been studied for decades, there is a lack of knowledge about diffusiondriven nucleation from gas cavities in a fluid flow. This paper presents comprehensive experimental data gained in a generic setup. The experiments indicate that the nucleation rate (frequency of bubble production) and the volume of the detaching bubbles show a non-linear dependence on the shear rate at the wall. Measurements of the growth of surface nuclei are in good agreement with theoretical considerations based on boundary layer theory. As far as we know, this paper is the most comprehensive experimental study of diffusion-driven nucleation and underlines the understanding of nucleation as self-excited cyclic process. The presented results might be used for the validation of axiomatic models or numerical simulations but also reveal that many questions still need to be answered. In future works it could be worthwhile to study different crevice geometries (squares, polygon, irregular shapes), other surface conditions (roughnesses, waviness) and the interaction of nucleation spots located close to each other.

Acknowledgements We would like to thank Prof. Dr.-Ing. F. Peters (Ruhr-Universität Bochum) for the valuable hints regarding the experimental setup. The authors thank S. Schlautmann from the MCS group, University of Twente for his support in the micro fabrication processes.

\section{References}

Andersen A, Mørch KA (2015) Cavitation nuclei in water exposed to transient pressures. J Fluid Mech 771:424448

Atchley AA, Prosperetti A (1989) The crevice model of bubble nucleation. J Acoust Soc Am 86(3):1065-1084

Bankoff SG (1958) Entrapment of gas in the spreading of a liquid over a rough surface. AIChE Journal 4(1):24-26

Bolanos-Jimenez R, Rossi M, Rivas DF, Kähler CJ, Marin A (2017) Streaming flow by oscillating bubbles: Quantitative diagnostics via particle tracking velocimetry. J Fluid Mech 820:529-548 
Borkent BM, Gekle S, Prosperetti A, Lohse D (2009) Nucleation threshold and deactivation mechanisms of nanoscopic cavitation nuclei. Phys Fluids 21(102003)

Bremond N, Arora M, Ohl CD, Lohse D (2005) Cavitation on surfaces. J Phys: Condens Matter 17(45)

Brennen CE (1995) Cavitation and Bubble Dynamics. Oxford engineering science series, Oxford University Press, New York

Brennen CE (2015) Cavitation in medicine. Interface Focus 5

Chen D, Pan LM, Ren S (2012) Prediction of bubble detachment diameter in flow boiling based on force analysis. Nucl Eng Des 243:263-271

Duhar G, Colin C (2006) Dynamics of bubble growth and detachment in a viscous shear flow. Phys Fluids 18(077101)

Epstein PS, Plesset MS (1950) On the stability of gas bubbles in liquid-gas solutions. J Chem Phys 18:1505-1509, DOI 10.1063/1.1747520

Fernandez Rivas D, Prosperetti A, Zijlstra A, Lohse D, Gardeniers HJGE (2010) Efficient sonochemistry through microbubbles generated with micromachined surfaces. Angew Chemie 122(50):9893-9895

Freudigmann HA, Iben U, Dörr A, Pelz PF (2017) Modeling of cavitation-induced air release phenomena in microorifice flows. J Fluids Eng 139(11)

Fritz W (1935) Berechnung des maximalvolumens von dampfblasen. Phys Z 36

Gelderblom H, Zijlstra AG, van Wijngaarden L, Prosperetti A (2012) Oscillations of a gas pocket on a liquid-covered solid surface. Phys Fluids 24(122101)

Groß TF, Pelz PF (2017) Diffusion-driven nucleation from surface nuclei in hydrodynamic cavitation. J Fluid Mech 830:138-164

Groß TF, Ludwig G, Pelz PF (2015) Experimental evidence of nucleation from wall-bounded nuclei in a laminar flow. In: Proceedings of CAV 2015: 9th International Symposium on Cavitation, Lausanne, Switzerland

Groß TF, Ludwig G, Pelz PF (2016) Experimental and theoretical investigation of nucleation from wall-bounded nuclei in a laminar flow. In: Proceedings of the 16th International Symposium on Transport Phenomena and Dynamics of Rotating Machinery, Honolulu, USA

Guzman DN, Hie Y, Chen S, Rivas DF, Sun C, Lohse D, Ahlers G (2016) Heat-flux enhancement by vapourbubble nucleation in rayleigh-bénard turbulence. J Fluid Mech 787:331-366

Henry W (1803) Experiments on the quantity of gases absorbed by water at different temperatures and under different pressures. Philos Trans R Soc London 93:29-274, DOI 10.1098/rstl.1803.0004

Jones SF, Evans GM, Galvin KP (1999a) Bubble nucleation from gas cavities - a review. Adv Colloid Interface Sci
$80: 27-50$

Jones SF, Evans GM, Galvin KP (1999b) The cycle of bubble production from a gas cavity in a supersaturated solution. Adv Colloid Interface Sci 80:51-84

Liger-Belair G (2004) Uncorked: The Science of Champagne. Princeton University Press, Princeton

Liger-Belair G (2005) The physics and chemistry behind the bubbling properties of champagne and sparkling wines: A state-of-the-art review. J Agric Food Chem 53(8):27882802

van der Linde P, Moreno Soto Á, Peñas-López P, RodriguezRodriguez J, Lohse D, Gardeniers H, van der Meer D, Rivas DF (2017) Electrolysis-driven and pressurecontrolled diffusive growth of successive bubbles on microstructured surfaces. Langmuir

Lochiel AC, Calderbank PH (1964) Mass transfer in the continuous phase around axisymmetric bodies of revolution. Chem Eng Sci 19:471-484

Lohse D, Zhang X (2015) Surface nanobubbles and nanodroplets. Rev Mod Phys 87(981)

Moreno Soto Á, Prosperetti A, Lohse D, van der Meer D (2016) Gas depletion through single gas bubble diffusive growth and its effect on subsequent bubbles. APS Division of Fluid Dynamics abstract D21.007

Nahra HK, Kamonati Y (2003) Prediction of bubble diameter at detachment from a wall orifice in liquid cross-flow under reduced and normal gravity conditions. Chem Eng Sci 58:55-69

Neumann TS (2002) Arterial gas embolism and decompression sickness. Physiology 77(2):77-81

Nüllig M, Peters F (2013) Diffusion of small gas bubbles into liquid studied by the rotary chamber technique. Chem Ing Tech 85:1074-1079

Parkin BR, Kermeen RW (1963) The roles of convective air diffusion and liquid tensile stresses during cavitation inception. In: Proceedings of IAHR Symp. on Cav. and Hyd. Mach., Sendai, Japan

Peñas-López P, Parrales MA, Rodriguez-Rodriguez J, van der Meer D (2016) The history effect in bubble growth and dissolution. part 1. theory. J Fluid Mech 800:180-212

Peñas-López P, Moreno Soto Á, Parrales MA, van der Meer and D (2017) The history effect in bubble growth and dissolution. part 2. experiments and simulations of a spherical bubble atached to a horizontal flat plate. J Fluid Mech 820:479-510

Peters F, Honza R (2014) A benchmark experiment on gas cavitation. Exp Fluids 55:1786

Prosperetti A (2017) Vapor bubbles. Annu Rev Fluid Mech 49:221-48

Rayleigh L (1879) On the capillary phenomena of jets. Proc R Soc Lond 29:71-97 
van Rijsbergen MX, van Terwisga TJC (2011) High-speed micro-scale observations of nuclei-induced sheet cavitation. In: WIMRC 3rd International Cavitation Forum 2011, Coventry, UK

Sarc A, Oder M, Dular M (2016) Can rapid pressure decrease induced by supercavitation efficiently eradicate legionella pneumophila bacteria? Desalination and Water Treatment 57(5)

Scriven LE (1959) On the dynamics of phase growth. Chem Eng Sci 10(1-2):1-13

Spiridonov EK (2015) Characteristics and calculation of cavitation mixers. Procedia Engineering 129:446-450

Verhaagen B, Fernandez Rivas D (2015) Measuring cavitation and its cleaning effect. Ultrasonics Sonochemistry 29:619-628

van Wijngaarden L (1967) On the growth of small cavitation bubbles by convective diffusion. Int J Heat Mass Tran 10(2):127-134

Zijlstra A, Fernandez Rivas D, Gardeniers HJGE, Versluis M, Lohse D (2015) Enhancing acoustic cavitation using artificial crevice bubbles. Ultrasonics 56:512-523 